\title{
Point-of-Care Platform Blood Biomarker Testing of Glial Fibrillary Acidic Protein versus S100 Calcium-Binding Protein B for Prediction of Traumatic Brain Injuries: A Transforming Research and Clinical Knowledge in Traumatic Brain Injury Study
}

\author{
David O. Okonkwo, ${ }^{1}$ Ross C. Puffer, ${ }^{1,2}$ Ava M. Puccio,, Esther L. Yuh, ${ }^{3-5}$ John K. Yue, ${ }^{4-6}$ \\ Ramon Diaz-Arrastia, ${ }^{7}$ Frederick K. Korley, ${ }^{8}$ Kevin K. W. Wang, ${ }^{9}$ Xiaoying Sun, ${ }^{10}$ Sabrina R. Taylor, ${ }^{4-6}$ \\ Pratik Mukherjee, ${ }^{3-5}$ Amy J. Markowitz, ${ }^{4-6}$ Sonia Jain,, ${ }^{10}$ and Geoffrey T. Manley ${ }^{4-6}$; \\ and the Transforming Research and Clinical Knowledge in Traumatic Brain Injury (TRACK-TBI) Investigators *
}

\begin{abstract}
Glial fibrillary acidic protein (GFAP) is cleared by the Food and Drug Administration (FDA) to determine need for head computed tomography (CT) within $12 \mathrm{~h}$ after mild traumatic brain injury (TBI) (Glasgow Coma Score [GCS] 13-15); S100 calcium-binding protein B (S100B) serves this function in Europe. This phase 1 biomarker cohort analysis of the multi-center, observational Transforming Research and Clinical Knowledge in Traumatic Brain Injury (TRACK-TBI) study compares GFAP's diagnostic performance, measured on a rapid point-of-care platform, against protein S100B to predict intracranial abnormalities on CT within $24 \mathrm{~h}$ post-injury across the spectrum of TBI (GCS 3-15). Head CT scan performed in TBI subjects and blood was collected for all consenting subjects presenting to 18 United States level 1 trauma centers. Plasma was analyzed on a point-of-care device prototype assay for GFAP and serum was analyzed for S100B. In 1359 patients with TBI (GCS 3-15), mean (standard deviation [SD]) age $=40.1$ (17.0) years; 68\% were male. Plasma GFAP levels were significantly higher in CT + TBI subjects (median $=1358 \mathrm{pg} / \mathrm{mL}$, interquartile range [IQR]: 472 3803) than in CT- TBI subjects (median $=116 \mathrm{pg} / \mathrm{mL}$, IQR: 26-397) or orthopedic trauma controls $(n=122$; median $=13 \mathrm{pg} / \mathrm{mL}$, IQR: 7-20), $p<0.001$. Serum S100B levels were likewise higher in CT+ TBI subjects (median $=0.17 \mu \mathrm{g} / \mathrm{L}$, IQR: $0.09-0.38$ ) than in CT- TBI subjects (median $=0.10 \mu \mathrm{g} / \mathrm{L}$, IQR: $0.06-0.18$ ), $p<0.001$. Receiver operating characteristic curves were generated for prediction of intracranial injury on admission CT scan; area under the curve (AUC) for GFAP was significantly higher than for S100B in the same cohort (GFAP AUC -0.85 , 95\% confidence interval [CI] $0.83-$ 0.87 ; S100B AUC $-0.67,95 \%$ CI $0.64-0.70 ; p<0.001)$. GFAP, measured on a point-of-care platform prototype assay, has high discriminative ability to predict intracranial abnormalities on CT scan in patients with TBI across the full injury spectrum of GCS 3-15 through $24 \mathrm{~h}$ post-injury. GFAP substantially outperforms S100B.
\end{abstract}

Keywords: biomarkers; glial fibrillary acidic protein; S100 calcium-binding protein B; traumatic brain injury

\footnotetext{
${ }^{1}$ Department of Neurological Surgery, University of Pittsburgh Medical Center, Pittsburgh, Pennsylvania, USA.

${ }^{2}$ Department of Neurological Surgery, Mayo Clinic, Rochester, Minnesota, USA.

${ }^{3}$ Departments of Radiology and ${ }^{6}$ Neurological Surgery, ${ }^{4}$ Brain and Spinal Injury Center, University of California, San Francisco, San Francisco, California, USA.

${ }^{5}$ Zuckerberg San Francisco General Hospital and Trauma Center, San Francisco, California, USA

${ }^{7}$ Department of Neurology, University of Pennsylvania, Philadelphia, Pennsylvania, USA.

${ }^{8}$ Department of Emergency Medicine, University of Michigan, Ann Arbor, Michigan, USA.

${ }^{9}$ Departments of Psychiatry and Neuroscience, McKnight Brain Institute, University of Florida, Gainesville, Florida, USA.

${ }^{10}$ Department of Family Medicine and Public Health, University of California San Diego, San Diego, California, USA.

*TRACK-TBI Investigators are listed after the Acknowledgments.
} 


\section{Introduction}

A CCORDING To the Centers for Disease Control (CDC), $\sim 4,800,000$ emergency department (ED) evaluations for traumatic brain injury (TBI) occur annually in the United States, and the number is increasing. ${ }^{1,2}$ The majority of patients $(80$ 90\%) presenting to the ED have mild TBI (Glasgow Coma Scale [GCS] score of 13-15), and in that group, $<10 \%$ will have abnormalities on head computed tomography (CT) and $<1 \%$ of those patients will require neurosurgical intervention. ${ }^{3,4}$

Blood tests to inform clinical practice have undergone intense study over the last two decades. Glial fibrillary acidic protein (GFAP) is one of the strongest candidate TBI diagnostic biomarkers in development. The clinical utility of GFAP as a TBI biomarker is most frequently compared against S100 calcium-binding protein B (S100B), as both are markers of glial injury and both are released into the bloodstream in response to injury in several pathological states, including TBI and other extracranial injuries. ${ }^{1,5-11}$ However, $\mathrm{S} 100 \mathrm{~B}$ is also found outside of the central nervous system, raising questions about its specificity as a TBI diagnostic biomarker, especially in patients with extracranial injuries. ${ }^{12}$

In 2007, an S100B blood test was added to the Scandinavian guidelines for non-severe brain injuries to reduce the number of CT scans and associated cost of evaluating mild TBI in the ED. ${ }^{13,14}$ Serum enzyme-linked immunosorbent assay (ELISA) measurement of GFAP in combination with ubiquitin C-terminal hydrolase L1 (UCH-L1) was cleared by the Food and Drug Administration (FDA) in 2018 for clinical use to identify mild TBI patients, defined as having a GCS score of 13-15, likely to have intracranial injuries on head CT in the acute phase $(<12 \mathrm{~h})$ after TBI. ${ }^{15}$ The S100B blood test to assess need for head CT is not approved in the United States. One of the barriers to clinical adoption of the current FDA-cleared GFAP biomarker is that it is a core laboratory test that can take up to $6 \mathrm{~h}$ to return a result. In addition, the narrow FDA indication of use does not include more severely injured TBI patients (GCS 3-12) or those who are evaluated $>12$ hours after injury.

A handheld, point-of-care (POC) device that rapidly $(<15 \mathrm{~min})$ measures TBI biomarkers on site would impact clinical practice globally. The current report is a pre-specified interim analysis of the prospective, multi-center, observational Transforming Research and Clinical Knowledge in Traumatic Brain Injury (TRACK- TBI) study. We evaluated a POC platform prototype assay (Abbott Laboratories) to measure plasma GFAP to predict intracranial abnormalities on head CT following trauma, and compared it against the European standard biomarker S100B. We sought to confirm and further support our previous GFAP findings ${ }^{16}$ by expanding the analysis to a much larger cohort, broadening the inclusion criteria to the full severity spectrum of TBI (GCS 3-15) and out to $24 \mathrm{~h}$ after injury, as well as comparing the diagnostic performance of plasma GFAP to that of serum S100B.

\section{Methods}

\section{Subjects and study design}

Subjects with TBI (GCS 3-15) were identified and enrolled prospectively in the TRACK-TBI study (TRACK-TBI; ClinicalTrials.gov: NCT02119182) as previously described. ${ }^{16,17}$ Subjects presenting to one of 18 participating level I United States trauma centers were enrolled from February 26, 2014 to July 27, 2018. The TRACK-TBI Phase 1 Biomarker Cohort $(n=1497)$ evaluated in this study was a pre-specified interim analysis that included the first half of the enrolled TBI subjects and controls (see subsequent text and Fig. 1). The study was approved by the institutional review board of each enrolling site. Written informed consent was obtained from subjects or their legal authorized representatives (LAR). Eligibility criteria included presentation within $24 \mathrm{~h}$ of injury with head trauma warranting clinical evaluation with a noncontrast head CT evaluation based on the 2008 American College of Emergency Physicians/Centers for Disease Control (ACEP/CDC) guidelines for neuroimaging and decision making in TBI. ${ }^{18}$ Subjects were eligible for inclusion as orthopedic trauma controls if they presented with isolated trauma to their limbs, pelvis, and/or ribs, and had an Abbreviated Injury Score (AIS) $<4$ for those body regions. Orthopedic trauma controls subjects were identified and enrolled using the same process as that for patients with TBI, except for the head CT requirement. Subjects were ineligible for enrollment as orthopedic trauma controls if they had loss of consciousness (LOC), disturbance of consciousness, post-traumatic amnesia (PTA)/retrograde amnesia, or other clinical findings suggestive of a brain injury.

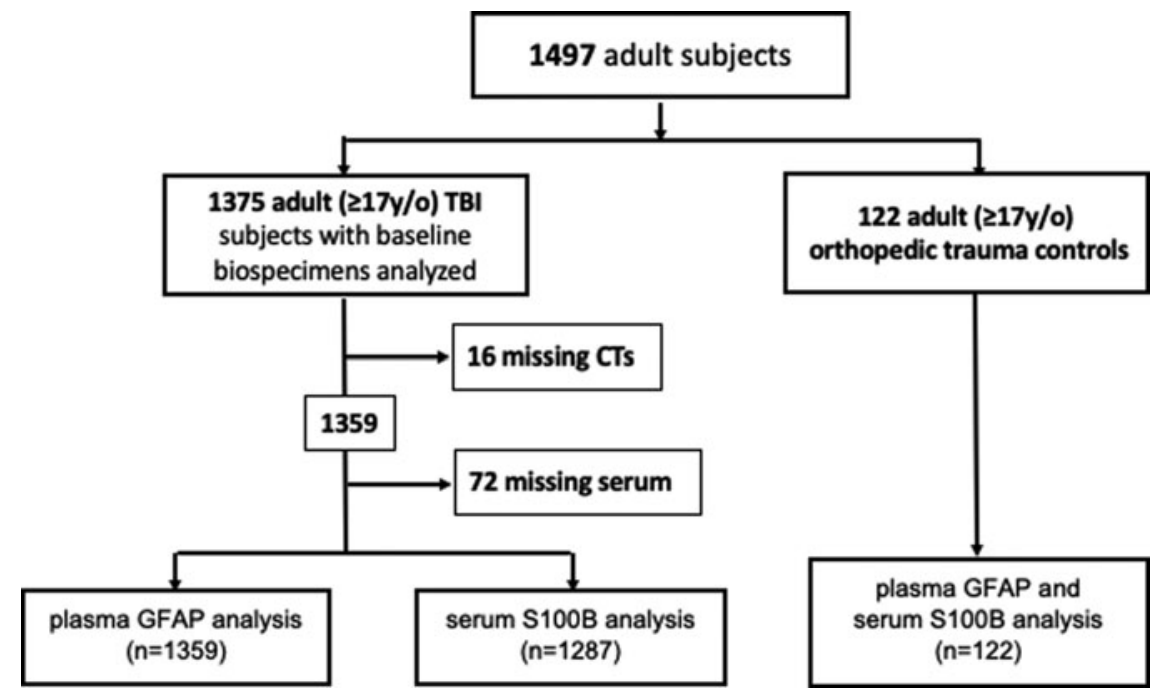

FIG. 1. Consolidated Standards of Reporting Trials (CONSORT) diagram of subjects from the prospective Transforming Research and Clinical Knowledge in Traumatic Brain Injury (TRACK-TBI) study included in current analysis. 


\section{Clinical data collection}

Demographic and clinical data, including GCS, source of trauma, LOC, and TBI diagnostic criteria were obtained at the time of initial evaluation by a treating physician and further confirmed by study staff through structured medical record review and subject or LAR interview when consent was obtained and blood samples collected.

\section{Sample collection and biomarker analysis}

Blood samples were collected from subjects who consented to genetic and proteomic analysis within a $24 \mathrm{~h}$ window from time of injury. All samples were dated and time stamped to confirm collection within the protocol's window. The TBI-Common Data Elements (CDE) Biospecimens and Biomarkers Working Group Guidelines for plasma preparation were followed (www.common dataelements.ninds.nih.gov/TBI.aspx). For detailed methods see TRACK-TBI Biorepository Manual of Procedures (https://tracktbi .ucsf.edu/sites/tracktbi.ucsf.edu/files/V4\%20TRACK-TBI\%20 Biospecimens\%20SOP\%205\%20MAY\%202016.pdf). Samples were centrifuged and aliquots stored at $-80^{\circ} \mathrm{C}$ for future batch processing. Sample storage occurred within $2 \mathrm{~h}$ of blood draw. Samples were batch-shipped as temperature-controlled express freight to the TRACK-TBI Biospecimen Repository at the University of Pittsburgh. All samples were stored in a de-identified manner, with a unique study number specific to site and subject. A central database was maintained by the coordinating center
(University of California at San Francisco [UCSF]) with each site entering site-specific data for final statistical reporting.

Sample analyses for GFAP and S100B were conducted in a blinded fashion. Ethylenediaminetetraacetic acid (EDTA) plasma was thawed in batches at room temperature and centrifuged at $10,000 \mathrm{rcf}$ for $10 \mathrm{~min}$ at $4^{\circ} \mathrm{C}$ prior to testing. GFAP concentrations were determined using prototype immunoassays on the i-STAT point-of-care platform (Abbott Laboratories, Abbott Park, IL). The i-STAT GFAP test uses the sandwich ELISA method with electrochemical detection of the resulting enzyme signal, which was optimized for plasma. The test time for each assay is $<15 \mathrm{~min}$. All samples were tested neat, without dilution, in duplicate. Samples reading greater than the calibration range were reported as greater than the reportable range and were not diluted.

The GFAP assay utilizes a monoclonal antibody for capture and a monoclonal antibody/alkaline phosphatase conjugate for detection of GFAP and GFAP breakdown products (BDPs). The GFAP calibrators range from 0 to $50,000 \mathrm{pg} / \mathrm{mL}$. The limit of detection (LoD) and limit of quantitation (LoQ) were determined using Clinical and Laboratory Standards Institute (CLSI) protocol EP17-A2 $2^{\mathrm{i}}$, are $<15 \mathrm{pg} / \mathrm{mL}$ and $<25 \mathrm{pg} / \mathrm{mL}$, respectively. Within-laboratory precision of $2.8-14.2 \%$ coefficient of variation $(\mathrm{CV})$ was demonstrated over a concentration range of $15,000-40 \mathrm{pg} / \mathrm{mL}$. Evaluation of assay linearity demonstrates $<10 \%$ deviation from linearity from 50,000 to $<25 \mathrm{pg} / \mathrm{mL}$, as defined by CLSI protocol EP6-A. ${ }^{19}$ In the analysis, when values were below the LoD, original values were used. Values $>50,000 \mathrm{pg} / \mathrm{mL}$ were imputed as $50,000 \mathrm{pg} / \mathrm{mL}$ in the analysis.

Table 1. TRACK-TBI Phase 1 Biomarker Cohort Demographics and Biomarker Analysis

\begin{tabular}{|c|c|c|c|c|}
\hline \multirow[b]{2}{*}{ Demographics } & \multicolumn{2}{|c|}{$T B I$} & \multirow[b]{2}{*}{ Orthopedic controls $(\mathrm{n}=122)$} & \\
\hline & $C T-(\mathrm{n}=810)$ & $C T+(\mathrm{n}=549)$ & & \\
\hline Age (years) & 37.7 (15.9) & 43.7 (17.9) & $39.2(15.0)$ & \\
\hline \multicolumn{5}{|l|}{ Mean (SD) } \\
\hline Range & $17-88$ & $17-90$ & $17-75$ & \\
\hline \multicolumn{5}{|l|}{ Sex } \\
\hline $\mathrm{F}$ & $294(36.3 \%)$ & $141(25.7 \%)$ & $43(35.3 \%)$ & \\
\hline M & $516(63.7 \%)$ & $408(74.3 \%)$ & $79(64.7 \%)$ & \\
\hline \multicolumn{5}{|l|}{ Cause of injury } \\
\hline RTA & $533(65.8 \%)$ & $282(51.7 \%)$ & $37(36.3 \%)$ & \\
\hline IF & $166(20.5 \%)$ & $170(31.1 \%)$ & $39(38.2 \%)$ & \\
\hline $\mathrm{V} / \mathrm{A}$ & $42(5.2 \%)$ & $55(10.1 \%)$ & $1(1.0 \%)$ & \\
\hline $\mathrm{O}$ & $69(8.5 \%)$ & $39(7.1 \%)$ & $25(24.5 \%)$ & \\
\hline \multicolumn{5}{|l|}{ Injury severity } \\
\hline GCS $13-15$ & $779(98 \%)$ & $358(74 \%)$ & $122(100 \%)$ & \\
\hline GCS 9-12 & $8(1 \%)$ & $40(8 \%)$ & - & \\
\hline GCS 3-8 & $8(1 \%)$ & $85(18 \%)$ & - & \\
\hline \multicolumn{5}{|l|}{ Prior TBI } \\
\hline Yes & $276(34.1 \%)$ & $118(21.5 \%)$ & $31(25.4 \%)$ & \\
\hline No & $534(65.9 \%)$ & $430(78.5 \%)$ & $91(74.6 \%)$ & \\
\hline \multicolumn{5}{|c|}{ Time to blood draw (h) ${ }^{\mathrm{a}}$} \\
\hline Mean (SD) & $13.1(6.8)$ & $16.1(5.8)$ & $13.3(7.3)$ & \\
\hline Biomarkers & $C T-$ & $C T+$ & Orthopedic controls & \\
\hline $\begin{array}{l}\text { GFAP }(\mathrm{pg} / \mathrm{mL}) \\
\text { Median (IQR) }\end{array}$ & $116(26-397)$ & $1358(472-3803)$ & $13(7-20)$ & $p<0.001$ \\
\hline $\begin{array}{l}\mathrm{S} 100 \mathrm{~B}(\mu \mathrm{g} / \mathrm{L}) \\
\text { Median (IQR) }\end{array}$ & $0.10(0.06-0.18)$ & $0.17(0.09-0.38)$ & $0.08(0.06-0.13)$ & $p<0.001$ \\
\hline
\end{tabular}

a Because of missing data, $n=796$ for CT- group; $n=540$ for CT + group.

CT, computed tomography; GCS, Glasgow Coma Score; GFAP, glial fibrillary acidic protein; IF, incidental fall; IQR, interquartile range; O, other; RTA, road traffic accident; S100B, S100 calcium-binding protein B; SD, standard deviation; TRACK-TBI, Transforming Research and Clinical Knowledge in Traumatic Brain Injury; V/A, violence/assault. 
Sample analysis for S100B was conducted by a single laboratory (University College of Dublin), in blinded fashion using an electrochemiluminescence immunoassay (Elecsys ${ }^{\circledR}$ S100B; Roche Diagnostics, Penzberg, Germany) on an automated Cobas ${ }^{\circledR}$ system from Roche. Serum samples were thawed in batches at room temperature and centrifuged at $10,000 \mathrm{rcf}$ for $10 \mathrm{~min}$ at $4^{\circ} \mathrm{C}$ prior to testing in duplicate. This assay is the trademarked assay used clinically in Europe for S100B (LoD: $<0.005 \mathrm{ug} / \mathrm{L}$; LoQ: not available per package insert; CV: intermediate precision of $20 \%$ ), which was optimized for serum. The full package insert is available publicly at https:// diagnostics.roche.com/no/en/products/params/elecsys-s100.html.

\section{CT scan evaluation}

As noted, eligible subjects had undergone a non-contrast head CT upon arrival in the ED as a part of their trauma evaluation in the clinical setting, and not specifically for research purposes. In the United States, the vast majority of clinical institutions follow the guidance set forth by the 2008 ACEP/CDC guidelines for neuroimaging and decision making in TBI. ${ }^{18}$ These CT scans were deidentified and uploaded to a central imaging database and then were graded based on recommendations of the neuroimaging working group of the TBI-CDE. ${ }^{20,21}$ Each reviewer was blinded to the identity and clinical information associated with a given CT scan. Head CT scans were classified as positive or negative based on the presence or absence of traumatic intracranial abnormalities (e.g., subarachnoid hemorrhage, subdural hematoma, contusion).

\section{Statistical analysis}

Demographics, clinical characteristics, and biomarker data were summarized and compared between TBI patients and orthopedic trauma controls, as well as among TBI patients with positive versus negative CT scans. Wilcoxon rank sum test was used for comparing continuous variables; Fisher's exact test was used for comparing categorical variables. The receiver operating characteristic (ROC)
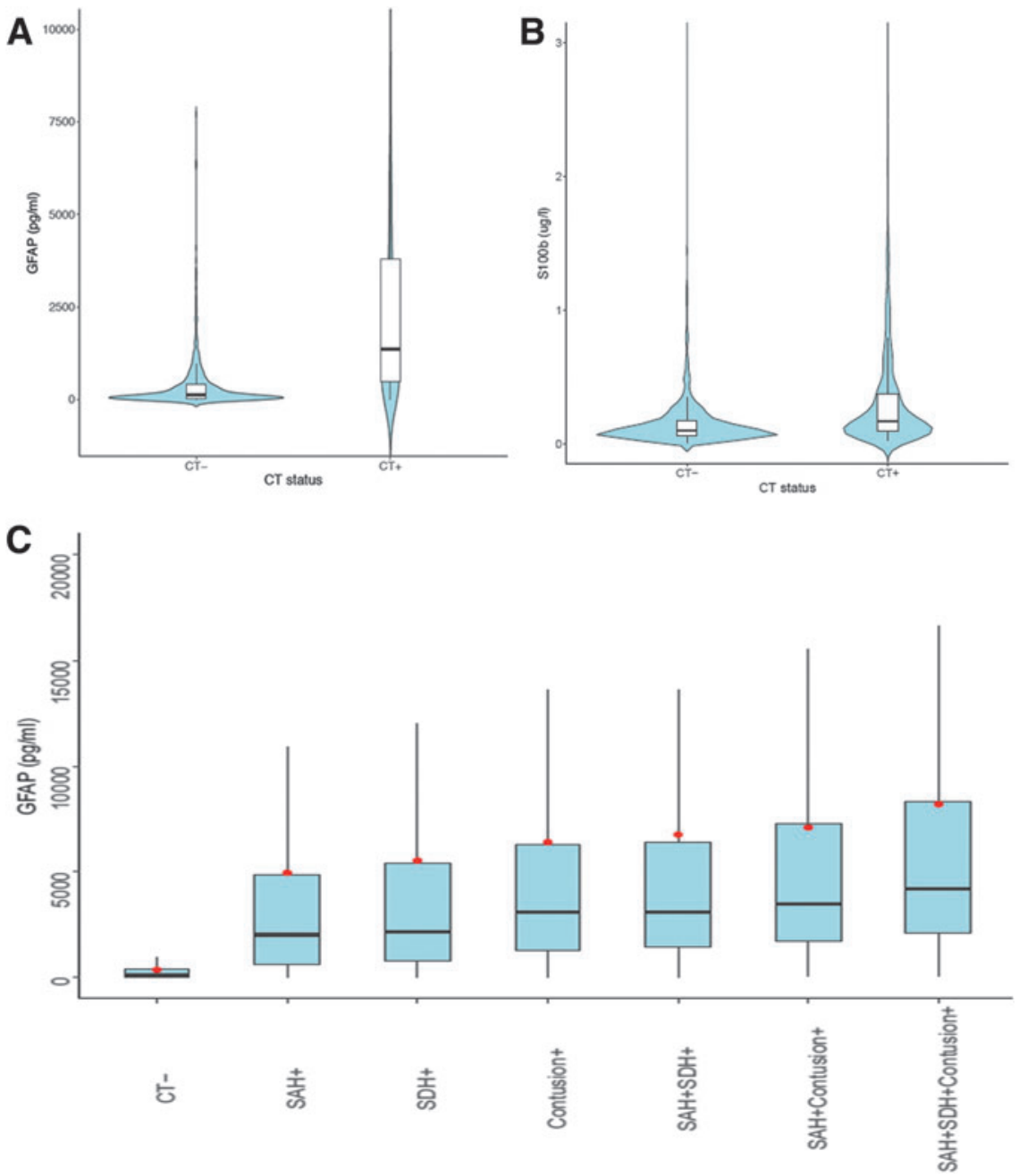

FIG. 2. Plasma glial fibrillary acidic protein (GFAP) and serum S100 calcium-binding protein B (S100B) levels by head computed tomography (CT) result across full spectrum of traumatic brain injury (TBI) (Glasgow Coma Score [GCS] 3-15). Violin plots of GFAP (A) and S100B (B) levels in TBI subjects (all GCS 3-15) with negative and positive head CT scans for intracranial injuries. Patients negative for intracranial injury on CT $(\mathrm{CT}-, n=810$ ) had a mean plasma GFAP $363.8 \mathrm{pg} / \mathrm{mL}$ (standard deviation [SD] $706.3 \mathrm{pg} / \mathrm{mL}$ ), whereas subjects positive for any intracranial lesion type $(\mathrm{CT}+, n=549)$ had a mean plasma GFAP level $3970.1 \mathrm{pg} / \mathrm{mL}$ $(\mathrm{SD} 7819.6 \mathrm{pg} / \mathrm{mL})$. Mean S100B levels were $0.181 \mathrm{ug} / \mathrm{L}(\mathrm{SD} 0.778 \mathrm{ug} / \mathrm{L})$ in the negative CT group $(n=753)$ and $0.405 \mathrm{ug} / \mathrm{L}(\mathrm{SD} 1.236$ $\mathrm{ug} / \mathrm{L})$ in the positive head CT group $(n=534)$. (C) Box plots of GFAP levels in plasma indicating higher levels of GFAP with progressively more severe intracranial injury spectra on head CT. Red dots indicate mean values. SAH, subarachnoid hemorrhage; SDH, subdural hematoma. Color image is available online. 
analysis was performed to determine whether GFAP can be used as a diagnostic tool for predicting head CT scan abnormality. The area under the ROC curve (AUC) was calculated with $95 \%$ confidence intervals (CI) and was compared with the AUC of S100B from the same patient sample via the bootstrap. The K-fold cross-validation method was used to select the optimal GFAP cutoff points for predicting CT abnormality based on the criteria of adjusted negative predictive value (NPV) reaching levels of $0.9,0.92,0.95$, and 0.98 . The prevalence rate for the adjusted NPV was estimated from the sample; 1000 bootstraps were conducted to determine the overall optimal cutoff using the median from each run. Then, the optimal cutoff points were applied to the full data to calculate the corresponding sensitivity, specificity, NPV, and positive predictive value (PPV). $P$ values $<0.05$ were considered statistically significant. Statistical analysis was conducted in $\mathrm{R}$, version 3.5.1 (R Core Team, 2013) by central study statisticians.

\section{Results}

A total of 1359 subjects with a head CT scan and blood samples drawn within the study protocol were eligible for inclusion in the current analysis. The Consolidated Standards of Reporting Trials (CONSORT) diagram for the study is presented in Figure 1. Plasma GFAP was measured in duplicate for all subjects. Serum S100B was analyzed in 1287 subjects. Orthopedic trauma control subjects had plasma GFAP and serum S100B analyzed $(n=122)$. The age of the
GFAP cohort (mean \pm standard deviation [SD]), was $40.1 \pm 17.0$ years, and 924/1359 (68.0\%) were male. Injuries sustained included $815(60.1 \%)$ road traffic accidents, $336(24.8 \%)$ incidental falls, 97 (7.2\%) episodes of violence or assault, and 108 (7.9\%) classified as "other" injuries. Ninety-three (7.3\%) subjects presented with severe TBI (GCS 3-8) on admission, 48 (3.8\%) presented with moderate TBI (GCS 9-12), and 1137 (88.9\%) presented with mild TBI (GCS 13-15) (Table 1). Loss of consciousness, post-traumatic amnesia, alteration of consciousness, and other demographic and clinical characteristics are presented in Supplementary Table S1.

Plasma GFAP levels (Table 1) were significantly higher in TBI subjects (median $=336 \mathrm{pg} / \mathrm{mL}$, interquartile range [IQR]: 69-1196) than in orthopedic trauma control subjects (median $=13 \mathrm{pg} / \mathrm{mL}$, IQR: 7-20), $p<0.001$. Serum S100B levels (Table 1) were likewise higher in TBI subjects (median $=0.12 \mu \mathrm{g} / \mathrm{L}, \mathrm{IQR}=0.07-0.24$ ) than in orthopedic trauma controls (median $=0.08 \mu \mathrm{g} / \mathrm{L}, \mathrm{IQR}=0.06-$ 0.13), $p<0.001$. In TBI patients, median GFAP level was $245 \mathrm{pg} / \mathrm{mL}(n=1137, \mathrm{IQR}=48-733)$ for GCS $13-15,2730 \mathrm{pg} / \mathrm{mL}$ $(n=48, \mathrm{IQR}=1624-4940)$ for GCS 9-12, and $4326 \mathrm{pg} / \mathrm{mL}(n=93$, $\mathrm{IQR}=1355-9171)$ for GCS 3-8. For S100B, these values were 0.11 $\mathrm{ug} / \mathrm{L}(n=1067, \mathrm{IQR}=0.07-0.18), 0.38 \mu \mathrm{g} / \mathrm{L} \quad(n=47, \mathrm{IQR}=0.19-$ $0.62)$, and $0.39 \mu \mathrm{g} / \mathrm{L}(n=92, \mathrm{IQR}=0.19-0.74)$, respectively.

We compared the diagnostic performance of GFAP versus S100B in predicting traumatic intracranial abnormality on head CT. In the GFAP cohort, 810 (59.6\%) subjects had a negative head

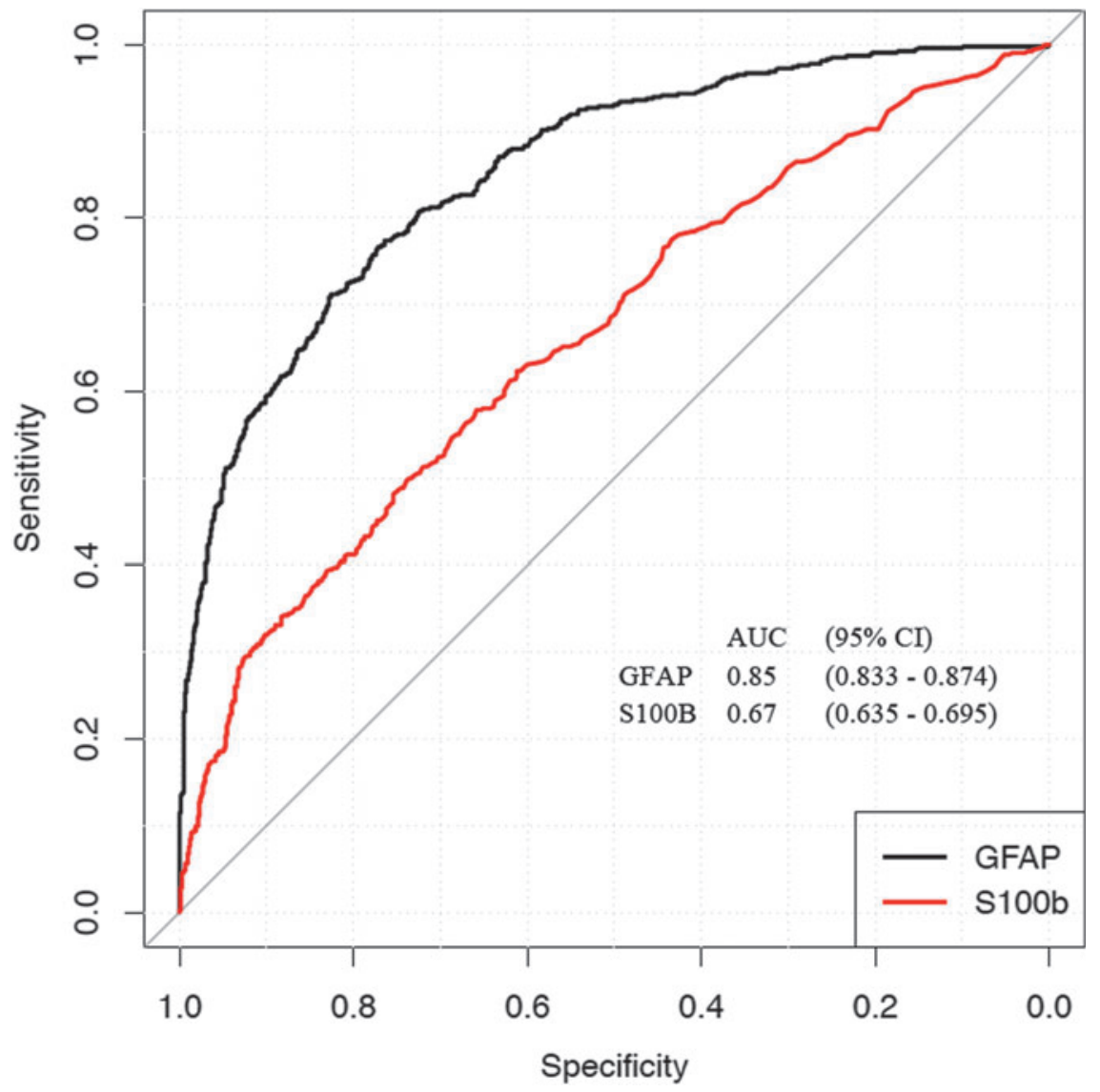

FIG. 3. Receiver operating-characteristic curve comparing performance of plasma glial fibrillary acidic protein (GFAP) and serum S100 calcium-binding protein B (S100B) as biomarkers for predicting intracranial injury on head CT scan within $24 \mathrm{~h}$ of injury across the full spectrum of traumatic brain injury (TBI) (Glasgow Coma Score [GCS] 3-15). The area under the curve (AUC) demonstrates that GFAP significantly outperforms S100B. GFAP measurements were performed on a point-of-care platform prototype assay capable of returning results within $15 \mathrm{~min}$. Color image is available online. 
TABle 2. AUCs (WITH 95\% CI) OF CT+ versus CT- by SAmpling Time Intervals

\begin{tabular}{lcc}
\hline & GFAP & S100B \\
\hline 0-6 h post-injury & $0.93(0.880-0.977)$ & $0.77(0.681-0.859)$ \\
7-12 h post-injury & $0.81(0.761-0.865)$ & $0.67(0.601-0.743)$ \\
13-18 h post-injury & $0.84(0.800-0.881)$ & $0.71(0.652-0.760)$ \\
19-24 h post-injury & $0.85(0.815-0.882)$ & $0.68(0.634-0.730)$ \\
\hline
\end{tabular}

AUC, area under the curve; CT, computed tomography; GFAP, glial fibrillary acidic protein; S100B, S100 calcium-binding protein B.

CT, whereas $549(40.4 \%)$ subjects had a positive CT scan. The mean time from injury to blood draw was $13.1 \mathrm{~h}(\mathrm{SD}=6.8)$ in subjects with a negative head CT, and $16.1 \mathrm{~h}(\mathrm{SD}=5.8)$ in subjects with a positive head CT. Plasma GFAP levels (Table 1, Fig. 2a) were significantly higher in subjects with a positive head CT (median $=1358 \mathrm{pg} / \mathrm{mL}$, IQR: 472-3803) than in those with a negative head CT $($ median $=116 \mathrm{pg} / \mathrm{mL}$ IQR: 26-397), and orthopedic trauma control subjects (median $=13 \mathrm{pg} / \mathrm{mL}$, IQR: 7-20), $p<0.001$. Serum S100B levels (Table 1, Fig. 2b) were higher in subjects with a positive head CT $($ median $=0.17 \mu \mathrm{g} / \mathrm{L}, \mathrm{IQR}=0.09-0.38)$ than in those with a negative head CT (median $=0.10 \mu \mathrm{g} / \mathrm{L}, \mathrm{IQR}=0.06-0.18$ ) and orthopedic trauma controls (median $=0.08 \mu \mathrm{g} / \mathrm{L}, \mathrm{IQR}=0.06$ $0.13), p<0.001$. Plasma GFAP measurements differed depending on the lesion type demonstrated on admission CT (subarachnoid hemorrhage $[\mathrm{SAH}]$, subdural hematoma $[\mathrm{SDH}]$, contusion), and were found to increase as the number of distinct traumatic intracranial lesions were identified (Fig. 2c). On subgroup analyses, CT+ patients within age (17-30, 31-40, 41-50, and 51-90 years), sex (male, female), and cause of injury (road traffic accident, incidental fall, violence/assault, other) cohorts had consistently statistically significantly higher GFAP and S100B values than CT- patients, with consistent AUCs across subgroups (data not shown).

ROC curves comparing GFAP and S100B for prediction of traumatic intracranial abnormality on head CT scan are presented in Figure 3. The AUC for GFAP was significantly higher than for $\mathrm{S} 100 \mathrm{~B}$ in the same participant cohort (GFAP AUC, $0.8595 \% \mathrm{CI}$ 0.83-0.87; S100B AUC, 0.67, 95\% CI 0.64-0.70), $\mathrm{p}<0.001$. By time of venipuncture, AUCs for GFAP values were 0.93 (CI $0.88-$ 0.98 ) for $0-6 \mathrm{~h}$ post-injury, 0.81 (CI $0.76-0.87$ ) for $7-12 \mathrm{~h}$ postinjury, 0.84 (CI $0.80-0.88$ ) for $13-18 \mathrm{~h}$ post-injury, and 0.845 (CI 0.82-0.88) for 19-24 h post-injury (Table 2). For S100B, the AUCs were 0.77 (0-6 h post-injury), 0.67 (7-12 h post-injury), 0.71 (13-18 h postinjury), and 0.68 (19-24 h post-injury) (Table 2) (see Supplementary Table S2 for additional details).

Cutoff levels of plasma GFAP for predicting traumatic intracranial abnormality on head CT in subjects with GCS 13-15 were selected based on the criteria of adjusted NPV reaching levels of $0.98,0.95,0.92$, and 0.90 , and their respective sensitivity, specificity, PPV, and NPV (Table 3).

\section{Discussion}

In this phase 1 biomarker cohort analysis of the prospective TRACK-TBI study, GFAP plasma measurement on a POC platform prototype assay was an excellent predictor of traumatic intracranial abnormalities on head CT scan when measured within $24 \mathrm{~h}$ of trauma. The POC assay produced a diagnostic performance comparable to commonly used blood tests in clinical practice for other disorders. ${ }^{22,23}$ GFAP substantially outperformed S100B as a TBI diagnostic biomarker of positive head CT scans. The current findings reaffirm the results of the Prospective Clinical Evaluation of Biomarkers of Traumatic Brain Injury (ALERT-TBI) trial for patients who present with a GCS of $13-15,{ }^{15}$ and support the introduction of a rapid, POC blood-based biomarker for clinical use in this population. They also suggest potential expansion of the current FDA test indication to include patients who present with a GCS $<13$, and to increase the FDA-indicated time of testing up to $24 \mathrm{~h}$ after injury.

With respect to predicting traumatic intracranial abnormalities on head CT scan, we observed a "dose response": the mean level of plasma GFAP increased with increasing numbers of distinct lesions identified on head CT. Plasma GFAP levels were also associated with the severity of the presenting GCS, with subjects in the severe to moderate range (GCS 3-12) having more than 10-fold higher GFAP levels than those with GCS 13-15. Although the recent FDA clearance for GFAP was for patients with GCS 13-15, our current results and prior studies indicate a role for identification of intracranial abnormalities on head CT in patients with an initial GCS $<13 .{ }^{16}$ Although it might be argued that GCS alone is sufficient for diagnosis and triage of these patients, there are many confounders of GCS, such as alcohol and drugs, where rapidly measured GFAP levels might facilitate earlier triage for a CT scan or reduce the number of CT scans performed in this population. ${ }^{16}$

The AUC of GFAP for predicting lesion on CT scan was 0.853 , falling into the "very good" performance category, as compared with the AUC of S100B (0.67, which equals poor performance) in the same cohort. Further, GFAP performed well in a more "realworld" cohort of TBI patients in the ED; namely, those further from $6 \mathrm{~h}$ from time of injury, as well as patients who have concomitant extracranial injuries. We found that GFAP AUCs continued to perform up to $24 \mathrm{~h}$ after injury, indicating that this POC platform prototype assay has utility for patients who present late for medical care, or for those who may have difficulty seeking immediate care, such as military personnel in combat situations. Our results also help to resolve the concern regarding the specificity of GFAP for TBI, especially in patients with extracranial injuries. ${ }^{24}$ We found that orthopedic trauma controls without TBI had significantly lower levels of GFAP, with median values that clearly distinguish them from subjects meeting clinical criteria for TBI, regardless of CT abnormalities. Although still able to discriminate between TBI subjects with CT abnormalities and orthopedic

Table 3. Performance of Plasma GFaP Cutoff Ranges for GCS 13-15 within 24 h of Injury ${ }^{\mathrm{a}}$

\begin{tabular}{lcccc}
\hline GFAP cutoff $(\mathrm{pg} / \mathrm{mL})$ & Sensitivity & Specificity & Positive predictive value & Negative predictive value \\
\hline 13.1 & $0.994(0.986,1)$ & $0.157(0.131,0.182)$ & $0.351(0.344,0.359)$ & $0.985(0.961,1)$ \\
37.8 & $0.964(0.944,0.980)$ & $0.303(0.271,0.340)$ & $0.389(0.377,0.402)$ & $0.949(0.921,0.973)$ \\
113.3 & $0.902(0.869,0.933)$ & $0.498(0.466,0.530)$ & $0.452(0.435,0.470)$ & $0.917(0.891,0.941)$ \\
190.1 & $0.846(0.810,0.883)$ & $0.594(0.561,0.630)$ & $0.490(0.466,0.515)$ & $0.894(0.872,0.917)$ \\
\hline
\end{tabular}

${ }^{a}$ Optimal cutoffs were selected based on the criteria of adjusted negative predictive value above a range of levels $(0.90,0.92,0.95,0.98)$ using the K-fold cross-validation method.

GFAP, glial fibrillary acidic protein. 
controls (median $0.17 \mu \mathrm{g} / \mathrm{L}$ vs. $0.08 \mu \mathrm{g} / \mathrm{L}$ ), the difference between TBI subjects without $\mathrm{CT}$ abnormalities and the controls was less distinct $(0.10 \mu \mathrm{g} / \mathrm{L}$ vs $0.08 \mu \mathrm{g} / \mathrm{L})$.

Using a predetermined GFAP cutoff value of $22 \mathrm{pg} / \mathrm{mL}$, the GFAP POC platform prototype assay had a sensitivity of 0.987 (95\% CI: 0.962-1) and an NPV of 0.988 (95\% CI: 0.959-1), supporting a potential clinical role for the test to rule out the need for a CT scan in patients with a history of TBI presenting to an emergency room. Although our finding compares favorably with the results of the ALERT-TBI trial, the current GFAP prototype POC assay will require development of new cutoff values specific to this device.

Our results validate a POC platform prototype GFAP assay that delivers results in minutes $(<15 \mathrm{~min})$ versus hours for the current FDA-cleared assay. With further clinical validation and FDA clearance of its use, this POC assay could be implemented for rapid assessment of GFAP in a variety of acute care settings such as the ED or a forward-deployed military setting, or in areas where core laboratory resources and CT scanning are sparsely available, such as low- and middle-income countries. Such a test has the potential to decrease unnecessary CT scans by $\geq 20 \%$, saving nearly $\$ 100$ million in medical expenses annually. ${ }^{17,18,25,26}$

\section{Limitations}

We recognize several limitations to this study. Subjects with a negative head CT may still meet the clinical criteria for TBI and therefore have elevated GFAP as compared with orthopedic trauma controls (median $116 \mathrm{pg} / \mathrm{mL}$ vs. $13 \mathrm{pg} / \mathrm{mL}$ ). We have found that $>25 \%$ of our subjects with a negative head CT have abnormalities on $3 \mathrm{~T}$ magnetic resonance imaging (MRI) consistent with TBI, ${ }^{27}$ and that those with a positive MRI have increased GFAP levels compared with controls. ${ }^{28}$ This suggests that in addition to helping triage which patients need a head CT, this test could provide a rapid means to screen patients as an aidin-diagnosis for TBI. Another limitation is that the majority of subjects in this study presented with mild TBI (GCS 13-15). Although this serves to confirm the findings from the ALERTTBI trial, it also suggests that patients with GCS $<13$ could benefit from earlier diagnosis of significant TBI, prior to the CT scan. This analysis was also limited to the adult TBI population. Further studies in the pediatric population are needed, given the morbidity and mortality of TBI in this population. ${ }^{1}$ We did not measure serial blood draws within the first $24 \mathrm{~h}$ to trend GFAP or S100B, which was beyond the scope of the current study and remains a topic for future projects focused on precision diagnostics. Although we have shown that GFAP is elevated even in CT-/MRI+ TBI compared with orthopedic controls without TBI ${ }^{28}$ further validation of GFAP in the TBI with polytrauma population, as well as in the field prior to arrival to $\mathrm{ED}$, is needed to augment the diagnostic indications of use for GFAP. This analysis is focused on glial markers of injury (GFAP and S100B); TBI also affects gray matter, and comparisons of neuronal markers of injury (e.g., UCHL-1, neuron specific enolase [NSE]) are the subject of a separate analysis. An additional limitation is that samples were collected, frozen, and batched for analysis on the POC platform. This may affect results, including identifying cutoff values and ranges that will change when the assay is run at the POC. A prospective study with real-time biomarker analysis on the POC platform is in the planning stages. Future analyses may also focus on the predictive ability of blood-based biomarkers such as GFAP and S100B for patients more likely to receive operative intervention.

\section{Conclusion}

GFAP, measured on a POC platform prototype assay, is a sensitive blood-based biomarker with high discriminative ability to predict intracranial injuries on CT scan in patients with TBI. GFAP substantially outperforms S100B. Our results support expansion of the current indication for GFAP to include more severely injured patients and a window of testing up to $24 \mathrm{~h}$ following trauma.

\section{Acknowledgments}

The authors thank Vibeke Brinck and Michael Jarrett (QuesGen Systems, Burlingame, CA, USA) for contributions to the development of the TRACK-TBI database.

\section{TRACK-TBI Investigators}

Opeolu Adeoye, Neeraj Badjatia, Kim Boase, Yelena Bodien, Ross Bullock, Randall Chesnut, John Corrigan, Karen Crawford, Sureyya Dikmen, Ann-Christine Duhaime, Richard Ellenbogen, Venkata Feeser, Adam R. Ferguson, Brandon Foreman, Raquel Gardner, Etienne Gaudette, Joseph Giacino, Dana Goldman, Luis Gonzalez, Shankar Gopinath, Rao Gullapalli, Claude Hemphill, Gillian Hotz, Joel Kramer, Natalie Kreitzer, Harvey Levin, Chris Lindsell, Joan Machamer, Christopher Madden, Alastair Martin, Thomas McAllister, Michael McCrea, Randall Merchant, Lindsay Nelson, Laura B. Ngwenya, Florence Noel, Eva Palacios, Daniel Perl, Miri Rabinowitz, Claudia Robertson, Jonathan Rosand, Angelle Sander, Gabriella Satris, David Schnyer, Seth Seabury, Mark Sherer, Murray Stein, Nancy Temkin, Arthur Toga, Alex Valadka, Mary Vassar, Paul Vespa, Ross Zafonte.

\section{Funding Information}

This work was supported by the following grants: National Institute of Neurological Disorders (NINDS) 1RC2NS069409-01, 3RC2NS069409-02S1, 5RC2NS069409-02, 1U01NS086090-01, 3U01NS086090-02S1, 3U01NS086090-02S2, 3U01NS08609003S1, 5U01NS086090-02, and 5U01NS086090-03; US Department of Defense (DOD) W81XWH-13-1-0441, and US DOD W81XWH-14-2-0176. In-kind support for blinded analyses of GFAP and S100 was provided by Abbott Laboratories. Patient travel and stipend expenses were supported by One Mind (Staglin Family and Gen. Peter Chiarelli).

\section{Author Disclosure Statement}

No competing financial interests exist.

\section{Supplementary Material}

Supplementary Table S1

Supplementary Table S2

\section{References}

1. Centers for Disease Control and Prevention (2016). Rates of TBIrelated emergency department visits, hospitalizations, and deaths United States, 2001-2010. https://www.cdc.gov/traumaticbraininjury/ data/rates.html (Last accessed April 2, 2020).

2. Korley, F.K., Kelen, G.D., Jones, C.M., and Diaz-Arrastia, R. (2016). Emergency department evaluation of traumatic brain injury in the United States, 2009-2010. J. Head Trauma Rehabil. 31, 379-387.

3. McMahon, P.J., Panczykowski, D.M., Yue, J.K., Puccio, A.M., Inoue, T., Sorani, M.D., Lingsma, H.F., Maas, A.I., Valadka, A.B., Yuh, E.L., Mukherjee, P., Manley, G.T., Okonkwo, D.O., and Investigators, T.-T. (2015). Measurement of the glial fibrillary acidic protein and its 
breakdown products GFAP-BDP biomarker for the detection of traumatic brain injury compared to computed tomography and magnetic resonance imaging. J. Neurotrauma 32, 527-533.

4. Smits, M., Dippel, D.W., Nederkoorn, P.J., Dekker, H.M., Vos, P.E., Kool, D.R., van Rijssel, D.A., Hofman, P.A., Twijnstra, A., Tanghe, H.L., and Hunink, M.G. (2010). Minor head injury: CT-based strategies for management-a cost-effectiveness analysis. Radiology 254, 532-540.

5. Lee, Y.B., Du, S., Rhim, H., Lee, E.B., Markelonis, G.J., and Oh, T.H. (2000). Rapid increase in immunoreactivity to GFAP in astrocytes in vitro induced by acidic $\mathrm{pH}$ is mediated by calcium influx and calpain I. Brain Res. 864, 220-229.

6. Stocchetti, N., Pagan, F., Calappi, E., Canavesi, K., Beretta, L., Citerio, G., Cormio, M., and Colombo, A. (2004). Inaccurate early assessment of neurological severity in head injury. J. Neurotrauma 21, $1131-1140$.

7. Vos, P.E., Lamers, K.J., Hendriks, J.C., van Haaren, M., Beems, T., Zimmerman, C., van Geel, W., de Reus, H., Biert, J., and Verbeek, M.M. (2004). Glial and neuronal proteins in serum predict outcome after severe traumatic brain injury. Neurology 62, 1303-1310.

8. Donato, R. (2001). S100: a multigenic family of calcium-modulated proteins of the EF-hand type with intracellular and extracellular functional roles. Int. J. Biochem. Cell Biol. 33, 637-668.

9. Donato, R., Sorci, G., Riuzzi, F., Arcuri, C., Bianchi, R., Brozzi, F., Tubaro, C., and Giambanco, I. (2009). S100B's double life: intracellular regulator and extracellular signal. Biochim. Biophys. Acta 1793, 1008-1022.

10. Steiner, J., Bernstein, H.G., Bielau, H., Berndt, A., Brisch, R., Mawrin, C., Keilhoff, G., and Bogerts, B. (2007). Evidence for a wide extraastrocytic distribution of S100B in human brain. BMC Neurosci. 8, 2.

11. Thelin, E.P., Nelson, D.W., and Bellander, B.M. (2017). A review of the clinical utility of serum S100B protein levels in the assessment of traumatic brain injury. Acta Neurochir. (Wien) 159, 209-225.

12. Haimoto, H., Hosoda, S., and Kato, K. (1987). Differential distribution of immunoreactive S100-alpha and S100-beta proteins in normal nonnervous human tissues. Lab. Invest. 57, 489-498.

13. Calcagnile, O., Anell, A., and Unden, J. (2016). The addition of S100B to guidelines for management of mild head injury is potentially cost saving. BMC Neurol. 16, 200.

14. Unden, J., Ingebrigtsen, T., Romner, B. and Scandinavian Neurotrauma Committee (2013). Scandinavian guidelines for initial management of minimal, mild and moderate head injuries in adults: an evidence and consensus-based update. BMC Med. 11, 50.

15. Bazarian, J.J., Biberthaler, P., Welch, R.D., Lewis, L.M., Barzo, P., Bogner-Flatz, V., Gunnar Brolinson, P., Buki, A., Chen, J.Y., Christenson, R.H., Hack, D., Huff, J.S., Johar, S., Jordan, J.D., Leidel, B.A., Lindner, T., Ludington, E., Okonkwo, D.O., Ornato, J., Peacock, W.F., Schmidt, K., Tyndall, J.A., Vossough, A., and Jagoda, A.S. (2018). Serum GFAP and UCH-L1 for prediction of absence of intracranial injuries on head CT (ALERT-TBI): a multicentre observational study. Lancet Neurol. 17, 782-789.

16. Okonkwo, D.O., Yue, J.K., Puccio, A.M., Panczykowski, D.M., Inoue, T., McMahon, P.J., Sorani, M.D., Yuh, E.L., Lingsma, H.F., Maas, A.I., Valadka, A.B., Manley, G.T., Transforming Research and Clinical Knowledge in Traumatic Brain Injury (TRACK-TBI) Investigators (2013). GFAP-BDP as an acute diagnostic marker in traumatic brain injury: results from the prospective transforming research and clinical knowledge in traumatic brain injury study. J. Neurotrauma 30, 1490-1497.

17. Yue, J.K., Vassar, M.J., Lingsma, H.F., Cooper, S.R., Okonkwo, D.O., Valadka, A.B., Gordon, W.A., Maas, A.I., Mukherjee, P., Yuh, E.L., Puccio, A.M., Schnyer, D.M., Manley, G.T., and TRACK-TBI Investigators (2013). Transforming research and clinical knowledge in traumatic brain injury pilot: multicenter implementation of the common data elements for traumatic brain injury. J. Neurotrauma 30, 1831-1844.

18. Jagoda, A.S., Bazarian, J.J., Bruns, J.J., Jr., Cantrill, S.V., Gean, A.D., Howard, P.K., Ghajar, J., Riggio, S., Wright, D.W., Wears, R.L., Bakshy, A., Burgess, P., Wald, M.M., Whitson, R.R., American College of Emergency Physicians, Centers for Disease Control and Prevention (2008). Clinical policy: neuroimaging and decisionmaking in adult mild traumatic brain injury in the acute setting. Ann. Emerg. Med. 52, 714-748.

19. Clinical and Laboratory Standards Institute (2012). Evaluation of Detection Capability for Clinical Laboratory Measurement Procedures; Approved Guideline, 2nd ed. Clinical and Laboratory Standards Institute: Wayne, PA.

20. Duhaime, A.C., Gean, A.D., Haacke, E.M., Hicks, R., Wintermark, M., Mukherjee, P., Brody, D., Latour, L., Riedy, G. and Common Data
Elements Neuroimaging Working Group Members, and Pediatric Working Group Members (2010). Common data elements in radiologic imaging of traumatic brain injury. Arch. Phys. Med. Rehabil. 91, 1661-1666.

21. Whyte, J., Vasterling, J., and Manley, G.T. (2010). Common data elements for research on traumatic brain injury and psychological health: current status and future development. Arch. Phys. Med. Rehabil. 91, 1692-1696.

22. Maisel, A.S., Krishnaswamy, P., Nowak, R.M., McCord, J., Hollander, J.E., Duc, P., Omland, T., Storrow, A.B., Abraham, W.T., Wu, A.H., Clopton, P., Steg, P.G., Westheim, A., Knudsen, C.W., Perez, A., Kazanegra, R., Herrmann, H.C., McCullough, P.A. and Breathing Not Properly Multinational Study Investigators (2002). Rapid measurement of B-type natriuretic peptide in the emergency diagnosis of heart failure. N. Engl. J. Med. 347, 161-167.

23. Reichlin, T., Hochholzer, W., Bassetti, S., Steuer, S., Stelzig, C., Hartwiger, S., Biedert, S., Schaub, N., Buerge, C., Potocki, M., Noveanu, M., Breidthardt, T., Twerenbold, R., Winkler, K., Bingisser, R., and Mueller, C. (2009). Early diagnosis of myocardial infarction with sensitive cardiac troponin assays. N. Engl. J. Med. 361, 858-867.

24. Posti, J.P., Hossain, I., Takala, R.S., Liedes, H., Newcombe, V., Outtrim, J., Katila, A.J., Frantzen, J., Ala-Seppala, H., Coles, J.P., Kyllonen, A., Maanpaa, H.R., Tallus, J., Hutchinson, P.J., van Gils, M., Menon, D.K., and Tenovuo, O. (2017). Glial fibrillary acidic protein and ubiquitin C-terminal hydrolase-L1 are not specific biomarkers for mild CT-negative traumatic brain injury. J Neurotrauma [Epub ahead of print].

25. Hunink, M.G. (2005). Decision making in the face of uncertainty and resource constraints: examples from trauma imaging. Radiology 235 , 375-383.

26. Ruan, S., Noyes, K., and Bazarian, J.J. (2009). The economic impact of S-100B as a pre-head CT screening test on emergency department management of adult patients with mild traumatic brain injury. $\mathrm{J}$. Neurotrauma 26, 1655-1664.

27. Yuh, E.L., Mukherjee, P., Lingsma, H.F., Yue, J.K., Ferguson, A.R., Gordon, W.A., Valadka, A.B., Schnyer, D.M., Okonkwo, D.O., Maas, A.I., Manley, G.T., and TRACK-TBI Investigators (2013). Magnetic resonance imaging improves 3-month outcome prediction in mild traumatic brain injury. Ann Neurol 73, 224-235.

28. Yue J.K., Yuh, E.L., Korley, F.K., Winker, E.A., Sun, X., Puffer, R.C., Deng, H., Choy, W., Chandra, A., Taylor, S.R., Ferguson, A.R., Huie, J.R., Rabinowitz, M., Puccio, A.M., Mukherjee, P., Vassar, M.J., Wang, K.K.W., Diaz-Arrastia, R., Okonkwo, D.O., Jain, S., Manley, G.T., and TRACK-TBI Investigators (2019). Diagnostic utility of plasma GFAP biomarker to identify MRI-positive findings among CT-negative traumatic brain injury patients using a prototype point-of-care platform: A TRACK-TBI cohort study. Lancet Neurol. 18, 953-61.

Address correspondence to: David O. Okonkwo, $M D, P h D$ Department of Neurological Surgery University of Pittsburgh Medical Center 200 Lothrop Street, Suite B-400 Pittsburgh, PA 15213

USA

E-mail: okonkwodo@upmc.edu

Amy J. Markowitz, JD

Brain and Spinal Injury Center University of California, San Francisco Zuckerberg San Francisco General Hospital and Trauma Center 1001 Potrero Avenue, Building, Room 101, Box 0899 San Francisco, CA 94143 USA

E-mail: amymarkowitz@gmail.com 